\title{
Impact of intelligent agents on the avoidance of spontaneous traffic jams on two-lane motorways
}

\author{
Jessica Malerczyk ${ }^{a}$, Sabine Lerch, Bernd Tibken and Anton Kummert \\ University of Wuppertal, School of Electrical, Information and Media Engineering, 42119 Wuppertal, Germany
}

\begin{abstract}
This paper approaches the evaluation of intelligent agents for the reduction and avoidance of spontaneous traffic jams, which arise without evident reason. Individual vehicles are regarded as intelligent agents that act autonomously. The basis of this work is the Nagel-Schreckenberg (NaSch) model. Its extensions by the velocity-dependent randomization (VDR) model and multiple lanes allow us to simulate realistic traffic and congestion situations on two-lane motorways. Our concept is applied to the model and analyzed by fundamental diagrams and the average velocity, for example. The results of this paper reveal that traffic congestions are avoided when using swarm intelligence in all vehicles since human behavior, especially misbehavior, is eliminated and the velocities determined by the intelligent vehicle are directly realized. Moreover, an amount of $30 \%$ of intelligent vehicles has a significantly positive impact on traffic flow.
\end{abstract}

\section{Introduction}

Climate change is a defining keyword of our time. The reduction of carbon dioxide emissions plays a fundamental role here. Compared to pre-industrial times before 1750 , $\mathrm{CO}_{2}$ concentration has increased by $40 \%$ due to human activity [1]. The main cause of greenhouse gas is the combustion of fossil fuels from which a significant amount is attributable to road traffic [2]. The reduction of vehicle emissions is, therefore, essential for climate protection. A high proportion of $\mathrm{CO}_{2}$ emissions are caused by traffic congestions.

In addition to traffic jams caused by accidents or construction sites, so-called phantom traffic jams often occur on motorways. The main reasons for them are overloaded roads and the resulting high traffic density. Then small disturbances, such as overreaction, sudden overtaking or dawdling, are sufficient to form a spontaneous traffic jam [3], [4].

The NaSch model is suitable for macroscopic traffic description by microscopic observation of individual vehicles. It is especially useful to simulate phantom traffic jams. In recent years the model has been widely used and adapted to real measurements, for example in [5], [6]. In addition to its use to investigate traffic flow, the model has also been used to examine different traffic phases and transitions [7]-[9]. The model can also be used to investigate approaches to congestion reduction. In our opinion, there is potential for further investigations, especially when considering phantom jams. For this purpose, on the one hand, the influence of vehicular ad hoc networks has been evaluated (VANETs) [10], on the other hand, the use of reinforcement learning has been analyzed
[11] in recent works. Other studies use the open-source traffic simulation package SUMO (Simulation of Urban Mobility) [12], [13], or a swarm-based approach [14].

As far as we know, the avoidance of phantom jams based on the extended NaSch model and the use of intelligent agents in different vehicle types have not yet been sufficiently simulated and investigated.

Here, this paper presents the suitability of intelligent vehicles for avoiding spontaneous traffic jams on motorways, taking into account the obligation to drive on the right. For this purpose, we have implemented the NaSch model and its extensions including human behavior. The application and potential of our concept are analyzed and evaluated using fundamental diagrams and the average velocity, for example.

In chapter 2, the NaSch model and its extensions as well as the functionality of intelligent vehicles we use in our approach is described. In addition, our implementation is presented. In chapter 3 , the results are exposed and discussed. The conclusion is revealed in chapter 4 .

\section{Approach}

\subsection{Nagel-Schreckenberg model}

The NaSch model is a cellular automaton for single-lane traffic. Space and time and also the velocities are discrete. The road is divided into cells that can either be empty or contain exactly one vehicle. The velocity of each vehicle $i$ at time $t$ is described by an inter value $v_{i}(t)=0,1,2 \ldots v_{\max , i}$, where $v_{\max , i}$ is the maximum velocity. In each time step, the following four rules are applied to all cars in parallel [15]:

\footnotetext{
a Corresponding author: malerczyk@uni-wuppertal.de
} 
(1) Acceleration

$$
v_{i}:=\min \left(v_{i}+1, v_{\max }\right)
$$

(2) Slowing down due to vehicles driving ahead

$$
v_{i}:=\min \left(v_{i}, d_{i}\right)
$$

(3) Randomization parameter for deceleration $v_{i}:=v_{i}-1$ with probability $p$ if $v_{i}>0$

(4) Vehicle motion

$$
x_{i}(t+1):=x_{i}(t)+v_{i}
$$

The first rule clarifies the desire of drivers to move at the maximum possible speed in order to reach their destination as fast as possible. The avoidance of accidents is modeled by the second rule, where $d_{i}$ is the distance to the next vehicle ahead. By using the probability $p$ in the third rule, the traffic model is adapted to real behavior, since no driver can maintain a constant velocity over a certain distance. The last rule defines the motion. A vehicle at position $x(t)$ at time $t$ is then moved forward by its recalculated velocity $v_{i}$ during the transition to the next time step $t \rightarrow t+1$.

\subsection{Velocity-dependent randomization model}

The NaSch model is not able to reproduce complex phenomena such as hysteresis or metastable states, as they are observed in real traffic situations. Within an extension, the VDR model, this behavior can be simulated [5]. In this model, the randomization parameter $p(v)$ depends on the velocity $v$, so that delayed moving off is modeled. In an initial step, this parameter is calculated as follows, where $p_{0}>p$ :

(0) Randomization parameter for deceleration

$$
p(v)=\left\{\begin{array}{cc}
p_{0}, & \text { if } v=0 \\
p, & \text { if } v \neq 0
\end{array}\right.
$$

After step (0), rules (1)-(4) of the NaSch model are executed.

\subsection{Two-lane model}

As we aim to investigate the formation of spontaneous traffic jams on motorways, we have to integrate a second traffic lane and introduce lane changing rules [6]. Here, asymmetric rules are used because, in many countries, it is only allowed to overtake on the left lane. In this case, a driver can change from the right to the left lane if the number of cells ahead on the same lane gap $_{r}$ is too low, compared to a chosen $d_{r}$, and the number of cells forward

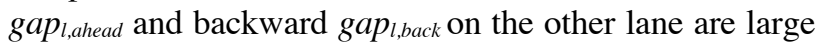
enough, compared to $d_{l, \text { ahead }}$ and $d_{l, b a c k}$, respectively. Furthermore, there is a lane change probability $p_{\text {change }}$, so that lane changes happen stochastically:

$$
\begin{gathered}
\operatorname{gap}_{r}<d_{r} \\
\operatorname{gap}_{l, \text { ahead }} \geq d_{l, \text { ahead }}
\end{gathered}
$$

$$
\begin{aligned}
\operatorname{gap}_{l, \text { back }} & \geq d_{l, \text { back }} \\
\operatorname{rand}() & <p_{\text {change }}
\end{aligned}
$$

Due to the obligation to drive on the right lane, a vehicle should change to the right, if possible, irrespective of the situation on the left lane. Therefore, the following three conditions are maintained, where the distances on the right lane gap $_{r, \text { ahead }}$ and gap $_{r, \text { back }}$ are taken into account and compared with $d_{r, \text { ahead }}$ and $d_{r, b a c k}$, respectively:

$$
\begin{gathered}
\operatorname{gap}_{r, \text { ahead }} \geq d_{r, \text { ahead }} \\
\operatorname{gap}_{r, \text { back }} \geq d_{r, \text { back }} \\
\text { rand }()<p_{\text {change }}
\end{gathered}
$$

A lane change takes place before the second rule of the $\mathrm{NaSch}$ model is applied, which can then be divided into two sub steps: First, a vehicle is moved in parallel but not forwards. Then the safety distance is maintained.

\subsection{Autonomous vehicles}

This paper investigates the influence of intelligent vehicles on the development of congestion. For this purpose, autonomous vehicles are assumed, which can be interpreted as agents within an intelligent swarm. They drive independently so that human intervention is not necessary. We suppose that the vehicles are equipped with the required sensors to precisely capture the environment, such as ultrasonic and radar sensors, cameras and lidar scanners, for example. That gives them accurate information about the distances to other vehicles and the corresponding velocities within a specific range.

\subsection{Implementation}

For the implementation in MATLAB, the enhanced NaSch model with a length of 400 cells is used. Each cell has a length of $7.5 \mathrm{~m}$ which is equal to the space a car occupies on average in a jam. A total number of 300 time steps are simulated where one time step is equal to $1 \mathrm{~s}$ and a closed system with a global density of $12 \%$ is used. The average values of 100 executed simulations are considered in the following chapter.

Each vehicle is initialized with a maximum possible velocity $v_{i, \max }$ between 3 and 7 cells per time step. It is not possible to accelerate beyond this value. With these different maximum velocities, we are able to model different types of vehicles, such as trucks and sports cars, for example. In this way, we achieve a more precise division of different vehicles than in other works, in which two vehicle types are considered [16], [17].

The velocities are initialized with a normal distribution with the expected value $E=5$ and the standard deviation $\sigma=2$. A velocity of $v=5$ is based on the recommended maximum velocity $v_{\text {rec }}=130 \mathrm{~km} / \mathrm{h}$ for German motorways. The corresponding real velocity to the value $v=0$ is $v_{\text {real }}=0 \mathrm{~km} / \mathrm{h}$ and to the value $v=7$ the real velocity is $v_{\text {real }}=189 \mathrm{~km} / \mathrm{h}$. Inside the integer interval $v=[0,7]$, all further real velocities are distributed linearly. 
Besides, each vehicle receives an individual lane change probability $p_{\text {change }}$ between 0.6 and 1.0 . This value differentiates between drivers who use every possible opportunity to change the lane and drivers who rather stay on their current lane. The randomization parameters for deceleration $p$ and $p_{0}$ are selected once between 0.1 and 0.3 and between 0.6 and 0.9 , respectively, at the beginning of the simulation. As soon as a vehicle needs more than four time steps to start, it is assigned $p$, which increases the likelihood to start in the following time step. In contrast to other approaches, we perceive this as more realistic modeling since in real traffic situations, other road users would alert the drivers who are not moving off.

A lane change to the left is considered if the number of free cells to the vehicle in front is less than its velocity value, compare (6). The conditions for a transition to the right lane are checked in each time step. The parameters for the conditions (7), (8) and (10), (11) are set to: $d_{l, \text { ahead }}=$ $3, d_{l, \text { back }}=4, d_{r \text {,ahead }}=v_{i}$ and $d_{r, \text { back }}=3$, where $v_{i}$ is the current velocity of the vehicle $i$. The aim is to simulate more realistic traffic in which vehicles change the lanes even though there is not always enough distance for the rear driver on the other lane to keep its previous velocity. In the applied model no passing zones are not considered.

Our simulated autonomous vehicles operate via two functions: On the one hand, the vehicles only change lanes if they do not force the vehicle approaching from behind to decelerate, neither in the current time step nor in the following time step. On the other hand, the vehicles are able to adapt to the vehicle in front and its driving behavior due to knowing its velocity in each time step.

\section{Results and discussion}

To analyze the influence of our concept, the model itself is first executed without autonomous vehicles. The resulting congestion situations are evaluated and a reference is obtained. With this reference, the results of the model using autonomous vehicles can be compared. For the purpose of generating the reference, the term congestion is defined. This describes the situation in which at least four vehicles in a row come to a standstill, equivalent to $v=0$.

\subsection{Intelligent vehicle rate of $100 \%$}

The use of fully automated vehicles is still a future vision and is considered as a significant research goal. Here, an autonomously driving vehicle replaces the human driver and, thus, eliminates human imperfections such as random behavior. Our results prove these assumptions: Not a single congestion situation is identified, when this concept is applied.

Regarding the average mean velocities in Figure 1, it can be seen that the velocity distribution shifts towards higher speeds. In addition, we can observe that no vehicle drives below a speed of $v=3$ on average. According to 2.5, this is the minimum initialization velocity. This only occurs due to the fact that no vehicle randomly reduces its speed. As a result, the vehicle behind does not have to decelerate and therefore does not decline beneath the limit of $v=3$. This implies that no traffic jams are able to occur.

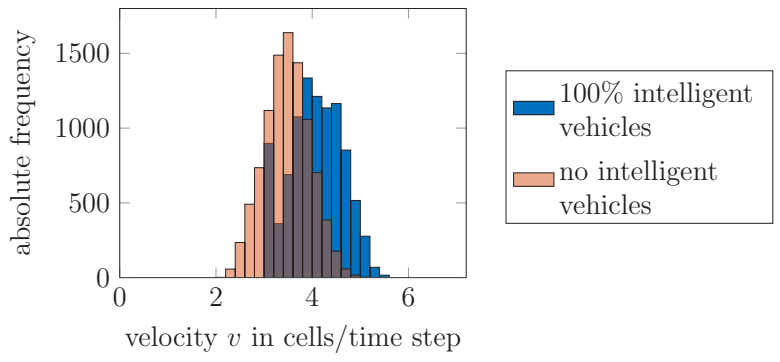

Figure 1. Histogram of average velocities from autonomous and non-autonomous vehicles

For further analysis of the traffic situation, we refer to Kerner's three-phase model, which subdivides traffic into three phases: free traffic $[\mathrm{F}]$, synchronized traffic $[\mathrm{S}]$ and wide moving jams [J] [18].

Figure 2 shows the fundamental diagrams of the reference on the one hand (top) and the applied concept on the other hand (bottom). Here only the diagrams of the right traffic lane are considered, because those for the left lane are very similar. In the upper diagram, the three phases are plainly visible. Both synchronized traffic and wide moving jam can be identified. In the lower diagram, however, there is only one phase, free traffic. The fact that there are no congested situations is visualized. The difference between the two diagrams, and thus the positive effect of intelligent vehicles is obvious.
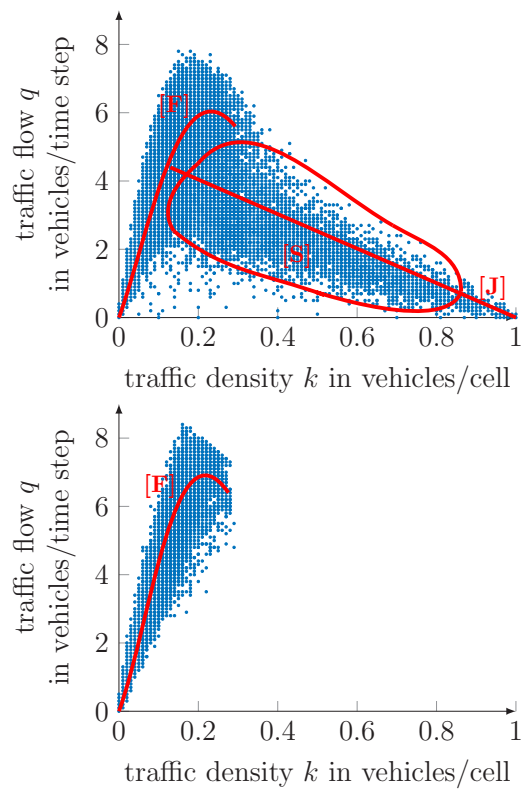

Figure 2. Fundamental diagrams of the reference (top) and of the applied concept, in which all vehicles are equipped with intelligent systems (bottom)

\subsection{Intelligent vehicle rate of $5 \%$}

Since a complete adoption of autonomous vehicles is very unlikely for the foreseeable future, we consider also the use of just a certain percentage of intelligent vehicles. That allows us to determine to what extent it is worth driving autonomously to avoid traffic jams. So, we combine both intelligent vehicles and human drivers in the simulations. 
We take [19] as a basis, which claims that a percentage of $5 \%$ of intelligent vehicles is sufficient to harmonize traffic. The analysis of our approach reveals that the number of identified traffic congestions increases by $19.8 \%$, which contradicts the statement in [19].

Over time, the number of traffic jams decreases continuously when no swarm intelligence is used. Considering the use of 5\% intelligent vehicles, the number increases initially, instead. Here, most traffic jams occur between the time steps 100 and 150, after which the amount of traffic jams lessens again. This is shown in Figure 3, where the left diagram shows the temporal occurrence of traffic congestion of the reference, and the right diagram illustrates those results when using 5\% swarm intelligence. A possible cause for this is the initialization. The vehicles are initialized in a way so they can maintain their initial speed in the first time step in any case. The comparatively few vehicles with integrated intelligent systems then adapt to the vehicles of their environment. They do not push or dawdle. After some time, human behavior predominates, resulting in dawdling or sudden overtaking. The comparatively small number of automated vehicles cannot compensate for this behavior. Traffic jams arise, but at a later point in time.
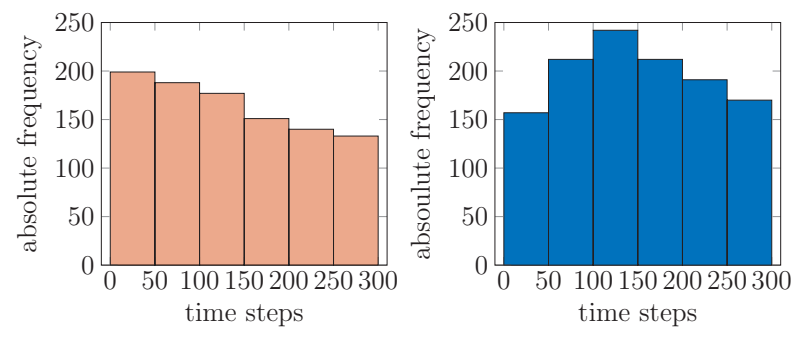

Figure 3. Histograms of time of origin of the identified traffic jams of the reference (left) and when using the concept with 5\% intelligent vehicles

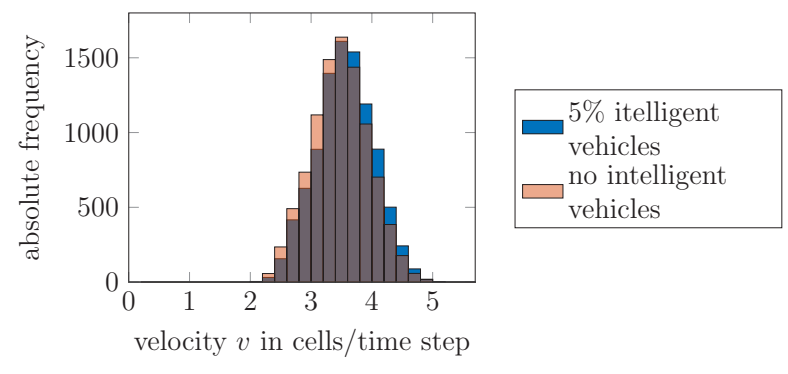

Figure 4. Histogram of average velocities from 5\% autonomous and non-autonomous vehicles

The maximum congestion width, as seen in Table 1, which means the number of vehicles involved in a jam, and the length of a congestion are further global characteristics we investigate. Considering these, a comparatively small positive effect caused by the few fully automated vehicles is observed. The comparison of average speeds shows a large similarity between our reference simulations and those with a $5 \%$ proportion of intelligent vehicles. This behavior is presented in Figure 4. Although shorter traffic congestions are observed, the increase in the number of traffic jams does not result in a significant difference of the velocity distribution. The vehicles are temporally shorter but more often involved in traffic congestions.

A comparison of the corresponding fundamental diagrams, here again using the example of the right-hand traffic lane, also shows no significant improvements in traffic flow when utilizing intelligent systems in $5 \%$ of all vehicles. The diagram in Figure 5 is almost identical with the upper diagram in Figure 2, which illustrates the results of our reference.

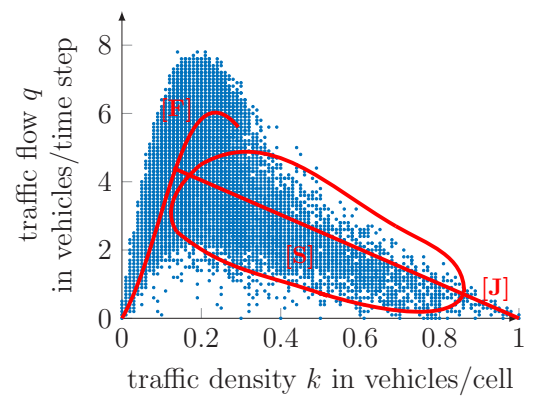

Figure 5. Fundamental diagram of the applied concept, in which $5 \%$ of all vehicles are equipped with intelligent systems

\subsection{Further intelligent vehicle rates}

In this respect, additional intelligent vehicle amounts of $10 \%, 20 \%, 30 \%, 40 \%$, and $50 \%$ are investigated. The observed results are presented in Table 1.

Evidently, the number and length of traffic jams decrease as the number of fully automated vehicles increases because they behave predictably. It is noticeable, however, that the maximum congestion width increases and therefore the number of vehicles involved. A possible cause could be convoys, which occur in about one-third of the simulations and are mainly caused by human drivers inside the simulation. Due to the imperfections of such drivers, individual dawdling vehicles may be sufficient to put the whole convoy into congested traffic flow. The tendency for a traffic jam to occur decreases, but if a traffic jam occurs, comparatively more vehicles are involved.

However, the fact that autonomous vehicles can start directly in the next time step means that these traffic jams dissolve more quickly. Furthermore, it can be observed that the average velocity of vehicles increases with a growing percentage of swarm intelligence. The reason for this is the lower number of traffic jams in which the vehicles lose their speed.

Another parameter studied in this paper is the ratio of average velocity to the maximum possible velocity of each vehicle. In addition to an undisturbed and comfortable journey, every driver also wishes to reach his destination as soon as possible. Obviously, this ratio also increases with an enhanced number of autonomous vehicles regarding the comparison.

Taking the results into account, we consider that a number of intelligent vehicles of around $30 \%$ has a positive impact on traffic flow. The amount of congestion situations shows a significant decrease. The length of traffic jams is reduced by more than a half and the average speed is increased significantly. 
Table 1. Overview of the influence of different ratios of autonomous and non-autonomous vehicles on traffic characteristics

\begin{tabular}{|l|c|c|c|c|c|c|c|c|}
\hline \multicolumn{1}{|c|}{ autonomous vehicle ratio } & $\mathbf{0 \%}$ & $\mathbf{5 \%}$ & $\mathbf{1 0 \%}$ & $\mathbf{2 0} \%$ & $\mathbf{3 0} \%$ & $\mathbf{4 0} \%$ & $\mathbf{5 0} \%$ & $\mathbf{1 0 0} \%$ \\
\hline \hline number of congestions in \% & 100 & +19.8 & +4.9 & -5.0 & -21.3 & -40.5 & -59.9 & -100 \\
\hline $\begin{array}{l}\text { temporal congestion length in time } \\
\text { steps }\end{array}$ & 56 & 39 & 32 & 22 & 20 & 18 & 16 & 0 \\
\hline $\begin{array}{l}\text { maximum congestion width in } \\
\text { vehicles }\end{array}$ & 7.6 & 7.5 & 7.5 & 7.3 & 7.7 & 8.0 & 8.3 & 0 \\
\hline average velocity in cells/time step & 3.4 & 3.5 & 3.5 & 3.6 & 3.7 & 3.7 & 3.8 & 4.0 \\
\hline $\begin{array}{l}\text { ratio of average to maximum } \\
\text { velocity in \% }\end{array}$ & 69.3 & 70.6 & 72.9 & 74.9 & 75.6 & 77.0 & 78.2 & 81.1 \\
\hline
\end{tabular}

\section{Conclusion}

The purpose of this paper is the evaluation of swarm intelligence to reduce and avoid spontaneous traffic jams on two-lane motorways. The basis was the extended NaSch model, which is suitable to model realistic traffic.

By knowing the distances and velocities to other vehicles within a certain range, autonomous vehicles were able to optimize the timing of their overtaking. This prevents the deceleration behavior of the vehicle behind. Besides, a predictive behavior with an orientation based on the velocity of the vehicle ahead has been implemented, resulting in more consistent motion.

At first, the influence of swarm intelligence implemented in all existing vehicles has been evaluated. The results reveal no congested situations. The intelligent vehicles calculate the time to a potential collision in each time step to adjust their speed accordingly. In this way, they prevent abrupt braking of their vehicle and, therefore, of the vehicle behind them, so that traffic congestions cannot occur. The average velocities increase and the traffic flow is undisturbed.

However, the use of completely autonomous driving vehicles for private purposes is currently not permitted due to the non-existing legal situation. The adoption of autonomous vehicles would proceed gradually. For this reason, the influence of swarm intelligence, installed in only $5 \%$ of all vehicles, was investigated secondly. However, the results show no positive impact compared to the reference, in which only human drivers were simulated, as human behavior predominates currently.

Only the use of intelligent vehicles in about one-third of all existing vehicles leads to a significant reduction of traffic jams and thus to a harmonization of traffic flow.

To summarize, our results show that future approval would have many advantages in terms of an improved traffic flow. Complete avoidance of congestions would not only lead to a faster arrival time at the destination, but also to a reduction in vehicle emissions.

\section{References}

1. P. Ciais, C. Sabine, G. Bala, L. Bopp, V. Brovkin, J. Canadell, A. Chhabra, R. DeFries, J. Galloway, M. Heimann and others, in Climate Change 2013: The Physical Science Basis, 465-570 (2014)
2. R. Sims, R. Schaeffer, F. Creutzig, X. Cruz-Núñez, M. D'Agosto, D. Dimitriu, M. J. F. Meza, L. Fulton, S. Kobayashi, O. Lah, A. McKinnon, P. Newman, M. Ouyang, J. J. Schauer, D. Sperling, G. Tiwari, in Climate Change 2014: Mitigation of Climate Change, 599-670 (2014)

3. G. Orosz, R. E. Wilson, R. Szalai, G. Stépán, Phys. Rev. E, 80, 046205 (2009)

4. Y. Sugiyama, M. Fukui, M. Kikuchi, K. Hasebe, A. Nakayama, K. Nishinari, S.-i. Tadaki, S. Yukawa, New J. Phys., 10, 033001 (2008)

5. R. Barlovic, L. Santen, A. Schadschneider, M. Schreckenberg, Eur. Phys. J. B, 5, 793-800 (1998)

6. M. Rickert, K. Nagel, M. Schreckenberg, A. Latour, Physica A, 231, 534-550 (1996)

7. R. Barlovic, T. Huisinga, A. Schadschneider und M. Schreckenberg, Phys. Rev E, 66, 046113 (2002)

8. W. Knospe, L. Santen, A. Schadschneider, M. Schreckenberg, J. Phys. A, 33, L477 (2000)

9. A. Schadschneider, Physica A, 285, 101-120 (2000)

10. F. Knorr, D. Baselt, M. Schreckenberg, M. Mauve, IEEE Trans. Veh. Technol., 61, 3490-3498 (2012)

11. S. Ishikawa, S. Arai, ICA (2016)

12. T. Liebig, M. Sotzny, COSIT (2017)

13. S. Djahel, N. Jabeur, R. Barrett, J. Murphy, ISNCC (2015)

14. C. Stolcis, E. Pfannerstill, IET INTELL TRANSP SYS, 11, 134-141 (2016)

15. K. Nagel, M. Schreckenberg, J. Phys., 2, 2221-2229 (1992)

16. D. Chowdhury, D. E. Wolf, M. Schreckenberg, Physica A, 235, 417-439 (1997)

17. W. Knospe, L. Santen, A. Schadschneider, M. Schreckenberg, Physica A, 265, 614-633 (1999)

18. B. S. Kerner, Phys. World J., 12, 25-30 (1999)

19. R. E. Stern, S. Cui, M. L. Delle Monache, R. Bhadani, M. Bunting, M. Churchill, N. Hamilton, H. Pohlmann, F. Wu, B. Piccoli and others, Transp. Res. Part C Emerg. Technol., 89, 205-221 (2018) 